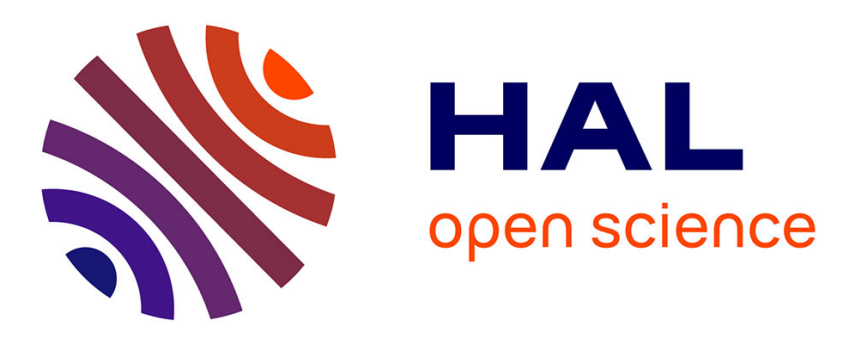

\title{
Investigation of high-order methods in large-eddy simulation of separated flow in a channel with periodic constrictions \\ Xavier Gloerfelt, Paola Cinnella
}

\section{- To cite this version:}

Xavier Gloerfelt, Paola Cinnella. Investigation of high-order methods in large-eddy simulation of separated flow in a channel with periodic constrictions. Direct and Large Eddy Simulation X, 24, Springer, pp.441-448, 2018, 978-3-319-63212-4. 10.1007/978-3-319-63212-4_56. hal-02877101

\section{HAL Id: hal-02877101 \\ https://hal.science/hal-02877101}

Submitted on 22 Jun 2020

HAL is a multi-disciplinary open access archive for the deposit and dissemination of scientific research documents, whether they are published or not. The documents may come from teaching and research institutions in France or abroad, or from public or private research centers.
L'archive ouverte pluridisciplinaire $\mathbf{H A L}$, est destinée au dépôt et à la diffusion de documents scientifiques de niveau recherche, publiés ou non, émanant des établissements d'enseignement et de recherche français ou étrangers, des laboratoires publics ou privés. 


\title{
Investigation of high-order methods in large-eddy simulation of separated flow in a channel with periodic constrictions
}

\author{
X. Gloerfelt and P. Cinnella
}

\section{Introduction}

In large-eddy simulations (LES), only the dynamics of large scales is computed and the effect of smaller scales is modelled. Scale separation is however difficult to establish since the low-pass filtering arises from a complex combination of implicit filtering by the grid and the discretization schemes. Even when explicit filtering is performed, the implicit filtering due to the application of discretization methods changes the shape of the filter function. The question of the intricate interactions between numerical errors, leading to this badly-defined filter, and subgrid-scale modelling errors has retained the attention of numerous authors since the studies of Ghosal [5] or Kravchenko and Moin [6]. Even with high-order schemes the subgrid contribution can be dominated by numerical errors in the high-wavenumber range. Meyers et al. [7] use error-landscapes to provide a more systematic assessment of computational errors. In explicitly-filtered LES, the filter shape plays a key role [10]. Since SGS models require informations from the smallest resolved scales, it is essential to ensure a high-accurate numerical approximation near the filter cutoff.

In the present study, several LES of the flow in a channel with streamwiseperiodic constrictions are presented. This is a particularly challenging benchmark case for the prediction of separation of turbulent flows from curved surface and subsequent reattachment. It has been designed to test LES or RANS/LES models $[4,9]$, and an ERCOFTAC database [8] is available for the hill geometry, reference LES and measurements. A large variety of numerical methods, meshing strategies, subgrid-scale (SGS) models, wall modelling assumptions (through either RANS

Xavier Gloerfelt

Arts et Métiers ParisTech, DynFluid Laboratory, Paris, France, e-mail: xavier.gloerfelte ensam.eu

Paola Cinnella

Università del Salento, via per Monteroni, Lecce, Italy \& Arts et Métiers ParisTech, DynFluid Laboratory, Paris, France, e-mail: paola.cinnella@ensam.eu 
equations or wall functions) have been investigated in the past $[3,4,9,11]$. The principal objective of the current study is to identify the sensitivity of the predictive accuracy to resolution and modelling issues. Simulations were performed for six different finite-difference schemes, six explicit filters and five subgrid-scale models on a coarse grid. It is shown in particular that the dissipative part of the discretization scheme plays a determinant role and overwhelms the choice of a SGS closure.

\section{Flow configuration and numerical strategy}

The chosen configuration corresponds to the streamwise-periodic-hill channel flow, as described in the ERCOFTAC website [8]. The Reynolds number based on the hill height and the bulk velocity at the top of the hill is $R e_{h}=10595$, and we choose a relatively low value for the Mach number, $M=0.2$. The dimensions of the domain are $L_{x}=9 h ; L_{y}=3.035 h ; L_{z}=4.5 h$, with $h$ the hill height. The upper and lower boundaries are isothermal solid walls, where no-slip velocity boundary conditions apply. Periodic boundary conditions are implemented in the streamwise $(x)$ and spanwise $(z)$ directions. A forcing function is used to impose a constant mass flux. The governing equations are the compressible Navier-Stokes equations written for a curvilinear domain by using a coordinate transformation. They are integrated in time using an explicit low-storage six-step Runge-Kutta scheme, optimised in the frequency space [2]. Space derivatives are approximated in the Cartesian regular computational space by using centered finite differences (FD), which are not dissipative. A filtering procedure is employed to remove spurious high-wavenumber oscillations. The filters have symmetric coefficients, and thus are non-dispersive. In the present work, several finite-difference and filtering schemes are tested to show the effect of the scheme resolvability on the quality of the LES simulations. A similar study has been done for a Taylor-Green vortex and a turbulent boundary layer [1]. Table 1 summarizes the different simulations discussed in the following.

The present LES strategy combines a finite-difference scheme with good spectral properties with the use of a selective filtering, which has many advantages. It prevents aliasing error and removes grid-to-grid oscillations. Moreover, the selective filtering provides the necessary regularization. The effect of SGS motions is taken into account implicitly in the high-wavenumber range thanks to the smooth truncations of the filter and finite-difference schemes in the wavenumber space. That is why this kind of approaches is considered as an implicit LES. In the following, the strategy is referred to as RT (for Regularization Term). The explicit-filter strategy can be used alone as in the RT strategy, or in conjunction with a SGS model. The first model tested is the classical Smagorinsky model supplemented by a van Driest damping function near the walls. Second, the dynamic version (DSM) with a Laplacian test filter is investigated. The dynamic constant is also filtered with a Laplacian to avoid too fast variations. Third, the multiscale model (MSM) is tested. Scale separation is performed by applying an 11-point filter with cutoff at $k \Delta x=\pi / 3$ [2]. In the small-small version (MSM-ss), both the SGS tensor and the eddy viscosity are 
Table 1 Summary of computational cases.

\begin{tabular}{|c|c|c|c|c|c|}
\hline Cases & Grid & $\begin{array}{l}\text { FD } \\
\text { scheme }\end{array}$ & Filter & SGS & Symbol/Line \\
\hline \multirow{3}{*}{ Grid convergence } & $64 \times 33 \times 32$ & DRP11 & DRP11 & RT & \multirow[t]{3}{*}{ black dash-dotted } \\
\hline & $128 \times 64 \times 64$ & DRP11 & DRP11 & RT & \\
\hline & $256 \times 128 \times 128$ & DRP11 & DRP11 & RT & \\
\hline \multirow{5}{*}{$\begin{array}{l}\text { Influence of } \\
\text { discretization } \\
\text { and filter order }\end{array}$} & $64 \times 33 \times 32$ & o2 & o2 & RT & \multirow{5}{*}{$\begin{array}{l}\text { grey dashed } \\
\text { grey solid } \\
\text { grey dash-dotted } \\
\text { black dashed } \\
\text { black solid }\end{array}$} \\
\hline & $64 \times 33 \times 32$ & 04 & 04 & RT & \\
\hline & $64 \times 33 \times 32$ & o6 & o6 & RT & \\
\hline & $64 \times 33 \times 32$ & o8 & o8 & RT & \\
\hline & $64 \times 33 \times 32$ & $\mathrm{o} 10$ & o10 & RT & \\
\hline \multirow{5}{*}{$\begin{array}{l}\text { Influence of } \\
\text { discretization } \\
\text { alone }\end{array}$} & $64 \times 33 \times 32$ & o2 & DRP11 & RT & \multirow{5}{*}{$\begin{array}{l}\text { grey dashed } \\
\text { grey solid } \\
\text { grey dash-dotted } \\
\text { black dashed } \\
\text { black solid } \\
\end{array}$} \\
\hline & $64 \times 33 \times 32$ & 04 & DRP11 & RT & \\
\hline & $64 \times 33 \times 32$ & 06 & DRP11 & RT & \\
\hline & $64 \times 33 \times 32$ & 08 & DRP11 & RT & \\
\hline & $64 \times 33 \times 32$ & o10 & DRP11 & RT & \\
\hline \multirow{5}{*}{$\begin{array}{l}\text { Influence of } \\
\text { filter order } \\
\text { alone }\end{array}$} & $64 \times 33 \times 32$ & DRP11 & o2 & RT & \multirow{5}{*}{$\begin{array}{l}\text { grey dashed } \\
\text { grey solid } \\
\text { grey dash-dotted } \\
\text { black dashed } \\
\text { black solid } \\
\end{array}$} \\
\hline & $64 \times 33 \times 32$ & DRP11 & o4 & RT & \\
\hline & $64 \times 33 \times 32$ & DRP11 & o6 & RT & \\
\hline & $64 \times 33 \times 32$ & DRP11 & o8 & RT & \\
\hline & $64 \times 33 \times 32$ & DRP11 & o10 & RT & \\
\hline \multirow{6}{*}{$\begin{array}{l}\text { Influence of } \\
\text { SGS modeling }\end{array}$} & $64 \times 33 \times 32$ & DRP11 & DRP11 & SM $C_{S}=0.18$ & \multirow{6}{*}{$\begin{array}{l}\text { circle/black solid } \\
\text { circle/black dashed } \\
\text { square/grey solid } \\
\text { square/grey dashed } \\
\text { diamond/black solid } \\
\text { diamond/black dashed }\end{array}$} \\
\hline & $64 \times 33 \times 32$ & DRP11 & DRP11 & $\mathrm{SM} C_{S}=0.1$ & \\
\hline & $64 \times 33 \times 32$ & DRP11 & DRP11 & $\mathrm{DSM} C_{d}$ & \\
\hline & $64 \times 33 \times 32$ & DRP11 & DRP11 & $\mathrm{DSM} C_{d} / 2$ & \\
\hline & $64 \times 33 \times 32$ & DRP11 & DRP11 & MSM-ls & \\
\hline & $64 \times 33 \times 32$ & DRP11 & DRP11 & MSM-ss & \\
\hline Ref. LES[8] & $280 \times 200 \times 220$ & $\mathrm{FV}^{a}$ & $\mathrm{FV}^{a}$ & DSM & white triangle/solid \\
\hline
\end{tabular}

${ }^{a} \mathrm{FV}$ : second-order finite volume (code LESSOC)

based on the filtered strain-rate tensor, whereas in the large-small version (MSM-ls), the SGS tensor alone is based on the filtered strain-rate tensor.

\section{Influence of numerical schemes}

The first investigation concerns the spatial discretization scheme on the coarsest grid. Two families of central finite-difference schemes are considered, namely standard second- to tenth-order schemes, denoted hereafter o2 to o10, and a DRP-type optimized scheme with an 11-point stencil [2] (DRP11). For brevity, only profiles along a vertical line at $x / h=2$, i.e. in the middle of the separated zone, are plotted in figure 1 . The first row of figure 1 shows the coupled influence of finite-difference and selective-filtering schemes. The separate role of the dispersive (FD) and dissipative (filter) parts are presented in the second and third row, respectively. Concerning the influence of the order of the standard finite-difference schemes, the mean flow profiles are affected by the high phase error introduced by the $2^{\text {nd }}$ and $4^{\text {th }}$-order 

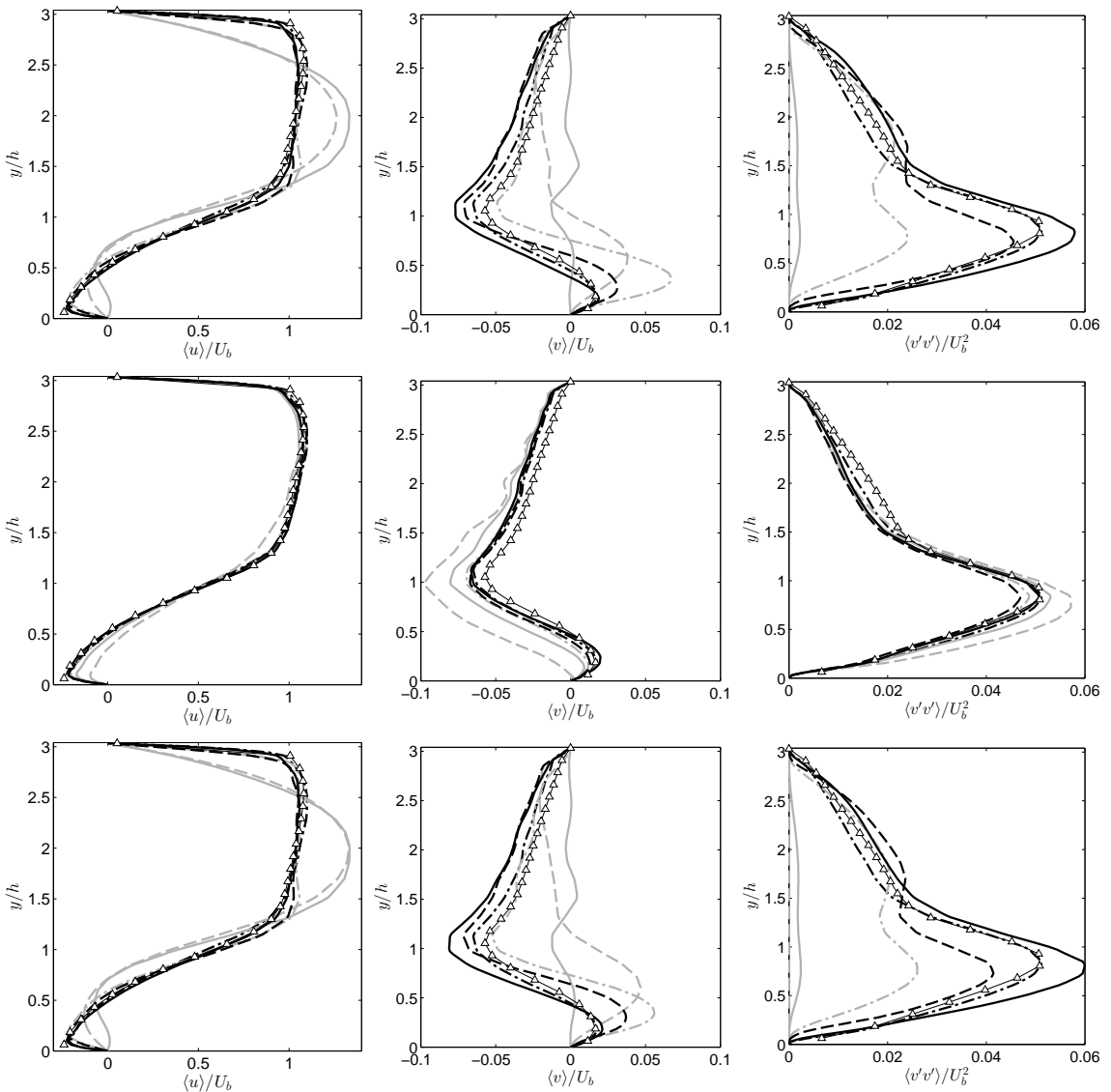

Fig. 1 From left to right, profiles of $\langle u\rangle ;\langle v\rangle$ and $\left\langle v^{\prime} v^{\prime}\right\rangle$ in the recirculation region at $x / h=2$. First row: coupled influence of finite-difference and selective-filtering schemes; second row: influence of finite-difference schemes supplemented by the DRP11 filter; third row: influence of selectivefiltering schemes along with the DRP11 scheme. Line legend is provided in Table 1.

scheme. The curves obtained beyond the $6^{\text {th }}$-order are rather similar, showing that the quality of the solution becomes independent of the spatial scheme order. The influence of the filter order in the RT method are shown in the second row of figure 1, where standard filters compared to the optimised filter and a reference LES [3]. The $2^{\text {nd }}$-order filter dissipates all of the turbulent structures. The $4^{\text {th }}$-order and $6^{t h}$-order filters are highly overdissipative. Contrary to the results obtained for finite differences schemes, a major influence is found up to the $10^{\text {th }}$-order for the filters. The similarity with the results of the first row, where the FD and filter schemes are changed simultaneously, indicates that the accuracy of the LES solution is highly dependent on the choice of a sufficiently sharp filter. The strong influence of the filter choice on the numerical resolvability is clearly illustrated in figure 2 for the instantaneous vorticity: using a sharper the filter allows capturing smaller structures. 
(a)

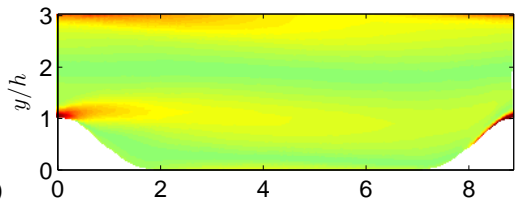

(c)
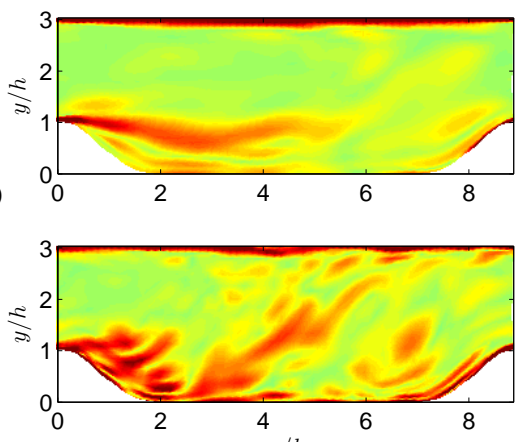

(e)

$x / h$
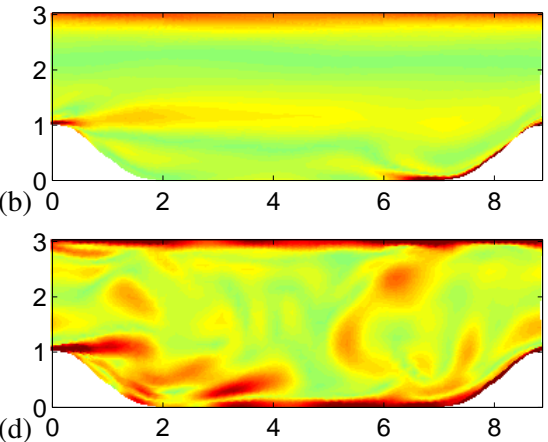

(d)

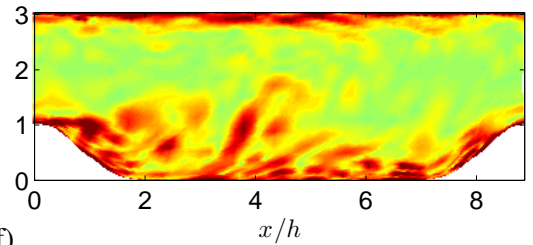

Fig. 2 Instantaneous snapshots of the vorticity norm: (a) FDo2/SFo2; (b) FDo4/SFo4; (c) FDo6/SFo6; (d) FDo8/SFo8; (e) FDo10/SFo10; (f) FD DRP11/SF DRP11.

\section{Influence of subgrid-scale models}

Results for several SGS models are presented in figure 3. This series of simulations uses the 11-point optimised finite-difference and filter schemes with $\chi=0.2$ (see Table 1). The Smagorinsky model with $C_{S}=0.18$, even in its dynamic form, appears to be too dissipative. As shown by several authors (e.g.[7]), the optimal values for the constant is lower. Taking $C_{S}=0.1$ or dividing by a factor of 2 the value of the dynamical constant yield results in fair agreement with the reference. In the same way, the use of multiscale models allows a significant improvement of the quality of the solution, but shows results similar to those obtained with the RT strategy alone. Overall the effect of the SGS model is weak and confirms that the numerical errors tend to be more influential than the SGS-model.

\section{Conclusion}

The separated flow in a channel with periodic constrictions is a good candidate to investigate the quality of LES.Coarse-grid LES have been conducted and show a fair agreement with the references for this benchmark case. It is shown that the dissipative part of the numerical discretization has a significant impact on the quality of the results. In particular its selectivity in the wavenumber space is of the utmost importance for increasing the resolved part of the dynamics. The influence of dif- 

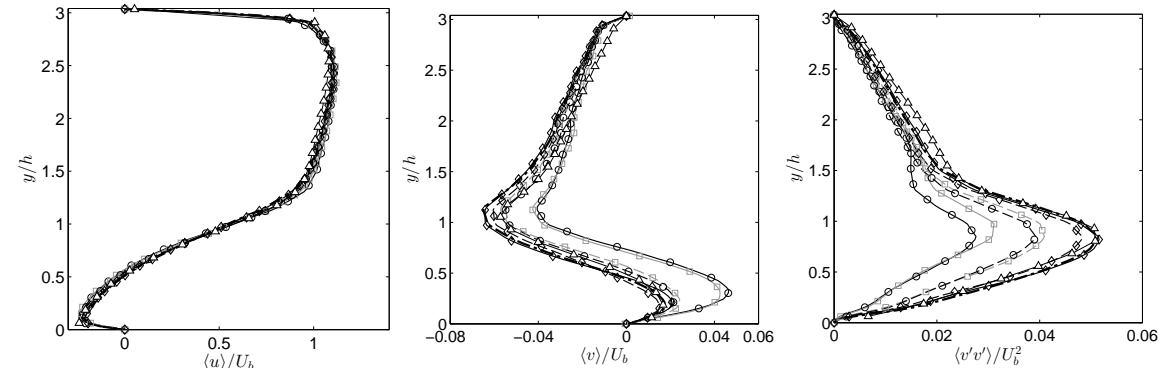

Fig. 3 From left to right, profiles of $\langle u\rangle$; $\langle v\rangle$ and $\left\langle v^{\prime} v^{\prime}\right\rangle$ in the recirculation region at $x / h=2$. Influence of subgrid-scale models. Line legend is provided in Table 1.

ferent SGS models has been tested and found to be relatively weak. This confirms that the numerical errors can dominate the role of the SGS-model.

Acknowledgements This work was granted access to the HPC resources of IDRIS and CCRT under the allocation 2014-1736 made by GENCI (Grand Equipement National de Calcul Intensif).

\section{References}

1. G. Aubard, P. Stefanin Volpiani, X. Gloerfelt, and J.-C. Robinet, Comparison of subgrid-scale viscosity models and selective filtering strategy for large-eddy simulations, Flow, Turbulence and Combustion, 91(3), 497-518 (2013).

2. C. Bogey and C. Bailly, A family of low dispersive and low dissipative explicit schemes for noise computation, J. Comput. Phys., 194, 194-214 (2004).

3. M. Breuer, N. Peller, C. Rapp, and M. Manhart, Flow over periodic hills - Numerical and experimental study in a wide range of Reynolds numbers., Computers and Fluids, 38vol. 38, pp. 433-457, 2009.

4. J. Fröhlich, C. Mellen, W. Rodi, L. Temmerman, and M. Leschziner, Highly resolved largeeddy simulation of separated flow in a channel with streamwise periodic constrictions," $J$. Fluid Mech., 526, 19-66 (2005).

5. S. Ghosal, An analysis of numerical errors in Large-Eddy Simulations of turbulence, J. Comput. Phys., 125, 187-206 (1996).

6. A. Kravchenko, and P. Moin, On the effect of numerical errors in Large Eddy Simulations of turbulent flows, J. Comput. Phys., 131, 310-322 (1997).

7. J. Meyers, P. Sagaut, and B. Geurts, Optimal model parameters for multi-objective large-eddy simulations, Phys. Fluids, 18, 095103 (2006).

8. C. Rapp, M. Breuer, M. Manhart, and N. Peller, 2D periodic hill flow. ERCOFTAC QNETCFD, http: //qnet-ercoftac.cfms.org.uk/w/index.php/UFR_3-30

9. Z. Xia, Y. Shi, R. Hong, Z. Xiao, and S. Chen, Constrained large-eddy simulation of separated flow in a channel with streamwise-periodic constrictions, J. Turbulence, 14(1), 1-21 (2013).

10. X. Yang, and S. Fu, The effect of filtering on truncated Navier-Stokes equations, J. Turbulence, 8(8), 1-18 (2007).

11. J. Ziefle, S. Stolz, and L. Kleiser, Large-eddy simulation of separated flow in a channel with streamwise-periodic constrictions, AIAA J., 46(7), 1705-1718 (2008). 